\title{
Estudio de experiencias inmersivas en museos. Las nuevas narrativas de la realidad aumentada
}

\author{
Adolfo Muñoz ${ }^{a}$, Ana Martí Testón ${ }^{b}$ \\ a,b Universitat Politècnica de València, ${ }^{\mathrm{a}}$ amunyoz@upvnet.upv.es, ${ }^{\mathrm{b}}$ anmartes@upv.es
}

\begin{abstract}
Resumen
En los últimos diez años hemos vivido una revolución en el ámbito de los museos y los sistemas de información. Desde 2015 nuestro grupo de investigación del Instituto de Diseño y Fabricación de la Universitat Politècnica de València investiga sobre la creación de experiencias inmersivas con gafas de realidad aumentada para la industria productiva y la cultural, investigando nuevas fórmulas que cambien radicalmente la manera en que nos relacionamos con los datos digitales en contextos inmersivos. En este artículo presentamos Holomuseum, una aplicación especialmente diseñada para crear exhibiciones de realidad aumentada para las gafas Hololens, y comparamos brevemente sus resultados con otras cuatro propuestas punteras en la aplicación de las tecnologías digitales en el ámbito expositivo: Back to Life del Natural History Museum de Londres; Survivors' Stories del Museo del Holocausto de Illinois; The Lost Palace del Whitehall Palace de Londres o la reconstrucción proyectada de la iglesia Románica de Sant Climent de Taüll de Lleida.
\end{abstract}

Palabras clave: museos, tecnología digital, realidad aumentada, experiencias inmersivas.

\section{Introducción}

En los últimos diez años los museos están viviendo una importante revolución gracias a la progresiva asimilación de las tecnologías de la información y la comunicación (TIC) por parte de los ciudadanos y las instituciones. La tecnología móvil, Internet y las redes wifi han posibilitado que los usuarios consulten los contenidos digitalizados a una velocidad impensable hace unos años, aprendiendo nuevas maneras de relacionarse con el patrimonio cultural, a la vez que adquieren nuevos conocimientos.

En este contexto, es cada vez más común ver como los museos utilizan aplicaciones para atender las necesidades particulares de los visitantes, y guiarles a través de recorridos 
personalizados, facilitando experiencias inmersivas e inolvidables. Es evidente que esta tendencia hacia la personalización de los contenidos, unida a la interpretación personal y a la interactividad, no se podría haber dado sin la ayuda de la implantación de últimos avances tecnológicos en nuestro entorno (Horwitz-Bennett, 2010) (Witcomb, 2003, pág. 122).

Pero pese a todos estos avances, todavía hoy es necesario analizar qué tipo de tecnologías son las más apropiadas para el contexto museístico, pues la tecnología per sé no será suficiente para ganar la atención del público, y cumplir así el cometido de hacer visible nuestra historia y nuestra cultura (Santacana \& Hernández, 2006). Se precisa además una nueva forma de narrar asequible y atractiva para la inmensa mayoría de la población, y especialmente atractiva para aquellos que nunca han visitado un museo. Narrativas adaptadas a los medios que nos ayuden a redescubrir el patrimonio cultural y poner en valor los fragmentos del pasado, con el fin de que formen parte de nuestro presente (Rico, 2006, pág. 152).

La tendencia de los últimos años ha sido, además, la utilización de las TIC y las redes sociales para promover la participación activa del ciudadano en el museo (Kelly, 2013), pues como ya dijo George Brown Goode ${ }^{1}$ en 1895, "los museos son lugares ideales dónde las audiencias y el personal pueden interactuar con el fin de crear y difundir el conocimiento al tiempo que atraen nuevos visitantes con sus ideas” (Goode, 1895, págs. 197-209). Además, la profusión de dispositivos móviles, como tabletas y teléfonos inteligentes, unida a la implantación de la Internet de banda ancha, ha facilitado que sea posible promover la participación de los visitantes también fuera del museo, aprovechando el auge de las redes sociales y su aceptación por la sociedad para difundir y publicitar sus actividades.

\section{Los dispositivos inmersivos y la necesidad de nuevas narrativas}

Además de la masificación de dispositivos móviles, en el año 2016 hemos vivido una nueva revolución comercial debida al principio de la implantación de los sistemas de realidad virtual y realidad aumentada en el mercado. Desde entonces, muchas gafas inteligentes están apareciendo y compitiendo para seducir a los consumidores.

Una de las ventajas más importantes de la realidad virtual como medio, que de momento no puede reproducirse mediante otro sistema, es que es capaz de colocar a las personas en el centro de una vivencia, por ejemplo, desde el punto de vista de otra persona diferente. En

\footnotetext{
${ }^{1}$ George Brown Goode fue uno de los primeros administradores de museos que publicó diversos textos relevantes y trabajó en el Smithsonian Institution.
} 
cambio, lo interesante de la realidad aumentada, a diferencia de la virtual, es que permite percibir lo recreado virtualmente sin perder la percepción del entorno real. Esto aporta seguridad y una manera diferente de entender y asumir la información digital (Osterhout, 2016).

Con la reciente aparición de los nuevos dispositivos de realidad aumentada (AR) del tipo "view through" o visión a través, se ha puesto de manifiesto la posibilidad de percibir e interactuar con contenidos virtuales en forma de hologramas anclados al espacio físico real. De hecho, en marzo de 2016 Microsoft presentó Hololens, unas gafas de realidad aumentada que se han popularizado por su capacidad para representar el mundo virtual sobre el mundo real mediante hologramas. Éstas incluyen un ordenador con múltiples sensores que permiten soportar aplicaciones con contenidos tridimensionales estereográficos ubicados en espacios reales, de manera que al usuario le parece que los objetos virtuales están incluidos en su espacio de visión natural.

Precisamente, uno de los retos más interesantes que nos ofrecen es la posibilidad de salir de la pantalla e interactuar con los datos digitales de una manera mucho más intuitiva, mediante comandos de voz y gestos. Lo que nos ofrece una puerta abierta hacia el futuro de la comunicación entre hombre y máquina, o, dicho de otra forma, entre el visitante de un museo y los datos digitales de las colecciones. La simbiosis de elementos reales y virtuales son capaces de generar nuevas narrativas donde lo virtual se superpone sobre lo real no para sustituirlo, sino para aumentar su fuerza comunicativa y emocional. Entendemos que este nuevo tipo de dispositivos de $\mathrm{AR}$, inaugurado con Hololens, se posiciona como un verdadero nuevo medio de comunicación, con infinitas posibilidades tanto en la industria como en el arte. Por ello, creemos que tendrán un papel destacado en los museos, abriendo el campo a infinitas posibilidades de desarrollo de narrativas asociadas a los elementos físicos del espacio expositivo que creen experiencias inmersivas memorables.

En nuestro empeño por aprovechar las nuevas capacidades de las gafas de AR, empezamos a desarrollar aplicaciones expositivas con el ánimo de testar nuevas narrativas asociadas a la unión de lo real y virtual en el mismo plano perceptivo, sabedores del reto que se presenta al tratarse de un nuevo medio de comunicación que está todavía por definir.

\section{Holomuseum, una aplicación para testar las exhibiciones de realidad aumentada en las salas de los museos}

Desarrollada en marzo 2017, Holomuseum es una aplicación pensada para crear exhibiciones de realidad aumentada para las gafas Hololens. El objetivo fue crear una aplicación que facilitara la ubicación y mantenimiento de una lista de objetos multimedia ubicados en internet, pero visibles en lugares determinados de la sala elegidos por el 
comisario de la exhibición. Al mismo tiempo este prototipo nos sirvió de banco de pruebas para probar los modos de interacción por gesto y por voz que admite el sistema, y hacer un estudio de usabilidad con los visitantes de la exposición que formó parte de una feria de los inventos de la UPV en julio de $2017^{2}$.

Holomuseum surgió de la necesidad de generar una aplicación específica para facilitar la creación de exhibiciones multipropósito que pudiesen demostrar las nuevas capacidades del medio de la realidad aumentada surgida con los dispositivos de "view through" como Hololens. Se trata de una herramienta preparada para satisfacer tanto las necesidades del comisario como del visitante.

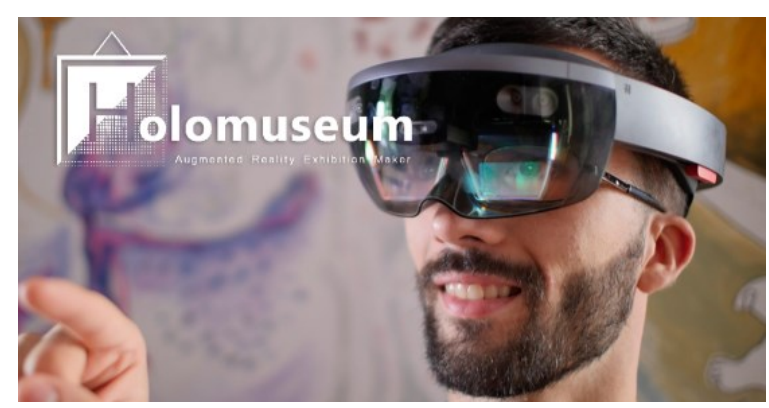

Fig.1 Ejemplo de usuario utilizando las gafas en Holomuseum. Fuente: Elaboración propia.

El sistema vino a solucionar varios problemas importantes: por una parte, aquellos surgidos por los propios límites actuales de la tecnología (manejo y mantenimiento de una colección de contenidos de virtuales en el espacio real y su ubicación estable en una o varias salas); y por otra, aquellos generados por la ausencia de un lenguaje de interacción consensuado para el manejo de contenidos de realidad aumentada con gafas holográficas y la creación de narrativas asociadas (Muñoz, 2017).

La aplicación se diseñó para ser utilizada en dos modos diferentes, uno para el comisario de la exposición y otro para el visitante. Por una parte, el comisario dispone del modo de edición para gestionar como curador la lista de objetos a exponer -objetos ubicados en un servidor web en forma de paquetes descargables durante la visita-, y se facilita la colocación de marcas virtuales en la sala que señalan la ubicación donde aparecerán los objetos cargados al ser solicitados por el visitante. Por otro lado, el modo del visitante, que es el modo en el que arranca la aplicación por defecto, está preparado para automatizar la carga y descarga de contenido a partir de la petición de la apertura de cajas por comandos

\footnotetext{
${ }^{2}$ Una descripción más detallada del proyecto se encuentra publicada en https://amunyoz.webs.upv.es/blog/holomuseum-unaaplicacion-para-crear-exhibiciones-de-realidad-aumentada/
} 
de voz, o gestos. Estas cajas virtuales se mostraban sobre carteles reales pegados en una pared de la sala, donde se explicaba brevemente su contenido y cómo operar sobre ellos.
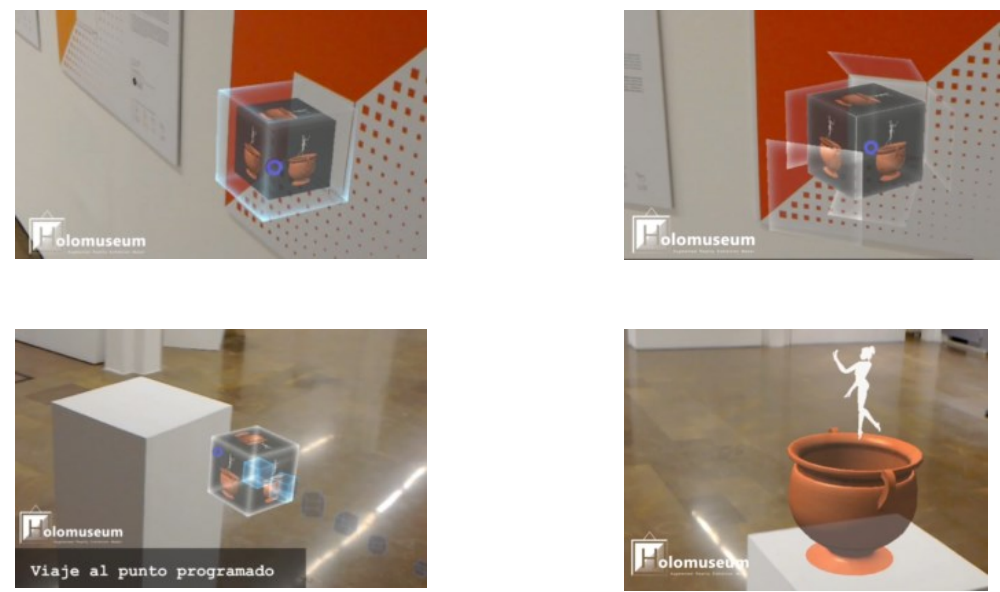

Fig.2 Ejemplos de interactividad de Holomuseum. 1: Caja activada descargando un contenido; 2: Caja abriéndose, junto antes de volar; 3: Caja viajando al punto programado de la sala; 4: El contenido aparece en el punto deseado. Fuente: Elaboración propia.

Con la idea de hacer una muestra de la usabilidad del sistema y las distintas posibilidades para crear narrativas asociadas, realizamos diferentes prototipos que se adecuaban a los distintos tipos de museos: por un lado, realizamos tres aplicaciones de estilo científico y tecnológico, y por otra parte tres de corte más artístico.

Respecto al diseño de la aplicación especialmente diseñada para los museos de corte más científico se decidió utilizar una explicación que incluía una diapositiva bidimensional que contenía elementos 3D animados con la intención de que ayudasen a facilitar la asimilación de conceptos difíciles de comprender en el espacio bidimensional.

En la experiencia de corte artístico utilizamos una narrativa inmersiva en la que se recreó una habitación entera, con elementos interactivos, y se invitó a los visitantes a pasear literalmente por el mismo. Esta narrativa permite realizar una visita similar a la experiencia tradicional del museo, en el sentido de que se basa en una disposición espacial natural, pero que incluye elementos interactivos y digitales.

En el estudio de usabilidad que realizamos se evidenció que ambas narrativas son aplicables a los distintos contextos, los visitantes quedaron satisfechos en su mayoría y estarían dispuestos a repetir e incluso pagar, por visitar exposiciones que utilizasen este tipo de tecnología. 


\section{Otras experiencias relevantes}

En relación con estas experiencias inmersivas, queremos comentar algunos casos relevantes realizados por otros museos internacionales para compararlas con nuestras experiencias y extraer algunas conclusiones.

Un ejemplo interesante es Back to Life, un proyecto desarrollado por Google Arts \& Culture en colaboración con el Natural History Museum de Londres (Google Arts and Culture, 2018) ${ }^{3}$, en el que utilizan la realidad virtual para que los fósiles del museo, de las bestias prehistóricas, cobren vida (Pavid, 2016). Según se puede observar al ponerse las gafas, o ver el vídeo en dispositivos móviles, el espacio del museo se llena de agua y un esqueleto colgado de la pared cobra vida, y flota, para pasar nadando justo delante de la mirada del espectador. Verdaderamente, creemos que este tipo de visualizaciones podrían ser mucho más impactantes si utilizasen los nuevos dispositivos de la realidad aumentada. Sería verdaderamente interesante ver in situ cómo el espacio del museo se llena de agua y los esqueletos cobran vida en la misma sala del museo.

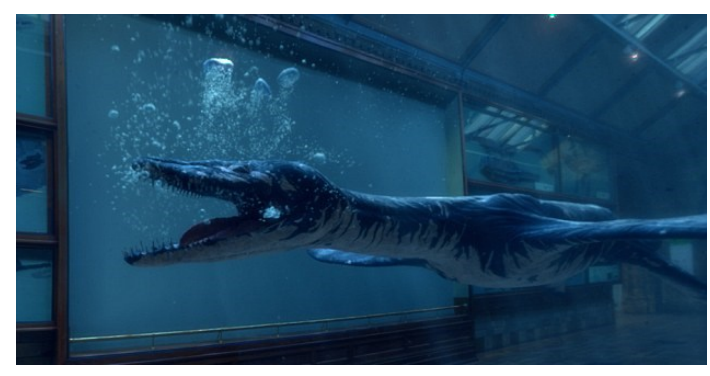

Fig. 3. Back to Life. El Rhomaleosaurus cobra vida virtualmente. Fuente: Google Arts and Culture (2016)

Acercándonos a las experiencias de inteligencia artificial y el uso de la realidad aumentada, queremos comentar Survivors' Stories, una experiencia realizada en el Museo del Holocausto de Illinois, en la que grabaron múltiples entrevistas a Sam Harris, un superviviente del holocausto, con aproximadamente 100 cámaras de video, mientras respondió alrededor de 1,200 preguntas en más de 20 horas de entrevista. El vídeo está grabado con croma y su imagen se proyecta como un holograma en el museo, al que los visitantes pueden hacer preguntas, y un software de reconocimiento de voz elige la respuesta adecuada (National Public Radio, 2017). Creemos que este tipo de experiencias

\footnotetext{
3 https://goo.gl/V37Kz9
} 
van a ser el futuro de las guías de muesos, y la inteligencia artificial se está utilizando ya para acercar los contenidos apropiados a cada visitante.

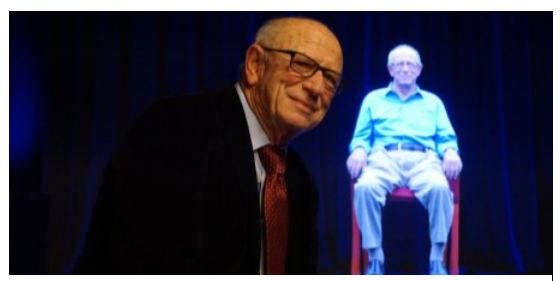

Fig. 4. Sam Harris en el Museo del Holocausto de Illinois.

Fuente: www.blog.deptagency.com

Otra propuesta interesante fue realizada el pasado 2013 en la iglesia Románica de Sant Climent de Taüll en Lleida, en colaboración con el MNAC, Museu Nacional d'Art de Catalunya de Barcelona. Las pinturas románicas de la iglesia han sido declaradas Patrimonio de la Humanidad por la UNESCO, pero por problemas de conservación, apenas son visibles en la actualidad. Por ello, un grupo de investigadores ha desarrollado un video mapping que recrea los frescos originales dentro del ábside mayor y el presbiterio de la nave central. La pintura original del siglo XII se conserva en el MNAC (Mapping Sant Climent de Taüll, 2013). Verdaderamente, las técnicas actuales de proyección audiovisual permiten devolver visualmente las pinturas al lugar que les corresponde mediante la fusión de las imágenes virtuales con las pinturas reales (Sierra, Riu-Barrera, Sugranyes, \& Pluma, 2015). Resulta interesante cómo logran un efecto de realidad aumentada que puede visualizarse sin la necesidad de utilizar dispositivos como las gafas, permitiendo además que se convierta en una experiencia mágica y colectiva, todavía no lograda con las gafas.

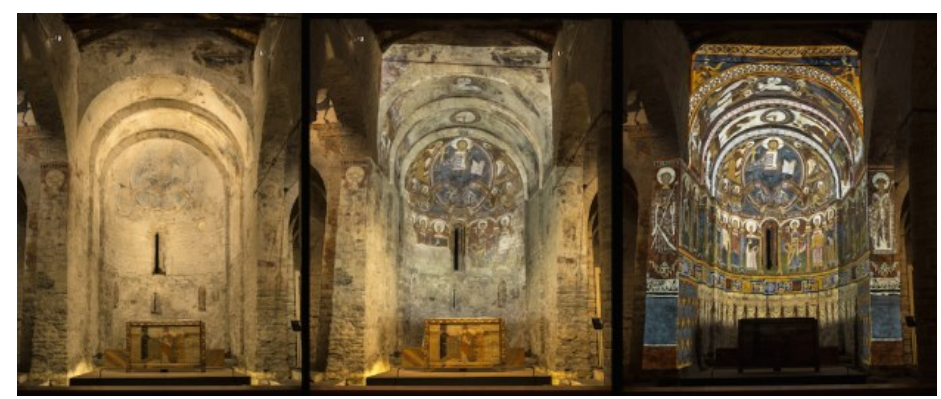

Fig. 5. Iglesia de Sant Climent de Taüll en el estado actual y la reconstrucción mediante video mapping. Fuente: MNAC (2013)

En este sentido, y yendo un paso más allá respecto a nuevas modalidades para lograr experiencias inmersivas mediante realidad aumentada, encontramos la propuesta de The Lost Palace, una exposición que se realizó utilizando audio teatro inmersivo (sonido binaural 3D y tecnología háptica) en el Whitehall Palace de Londres, para guiar a los 
visitantes en un viaje aumentado a través de la historia. Los usuarios llevan un aparato recubierto de madera conectado a unos auriculares. En el interior del aparato instalaron un smartphone, que interactuaba con las etiquetas NFC ubicadas en las instalaciones, lo que permitía escuchar, tocar y sentir el pasado (Richardson, 2017) (Heritage in motion, 2017). La colaboración entre diferentes industrias creativas dio buen resultado y, finalmente, lo que podría haber sido otra aplicación de museo poco llamativa, se convirtió en algo

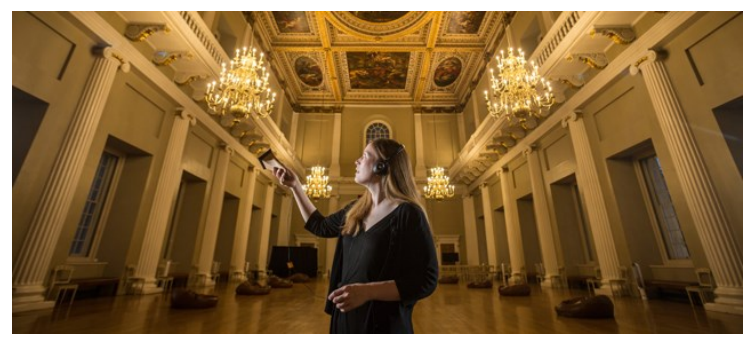

Fig. 6. The Lost Palace, una exposición que se realizó utilizando audio teatro inmersivo en el Whitehall Palace de Londres.

Fuente: creators.vice.com

realmente memorable e inmersivo (Richardson, 2017). Este ejemplo nos demuestra como la utilización de la tecnología innovadora no depende únicamente de las últimas novedades tecnológicas, sino de la capacidad de desarrollar narrativas interactivas e inteligentes que ayuden a dar vida al patrimonio.

\section{Conclusiones}

Hemos podido comprobar cómo los museos están investigando cada vez más nuevas formas de contar historias, de involucrar a las audiencias y entretenerlas, al mismo tiempo que atienden a las necesidades específicas de un perfil de visitante cada vez más heterogéneo. Las diferentes tecnologías digitales nos proporcionan nuevas oportunidades al tiempo que también plantean nuevos retos. Por un lado, los dispositivos móviles como los teléfonos inteligentes o las tabletas se presentan como herramientas interesantes ya que son personales y portátiles y, a menudo, están disponibles, ya que la mayoría de los visitantes utilizan sus propios dispositivos dentro del museo. Por otro lado, estos mismos dispositivos pueden convertirse en un obstáculo que perturbe la experiencia de la visita, limitando la interacción social y física con otros visitantes y afectando al modo en que se percibe la información y los objetos originales.

Últimamente, además, se han introducido otros dispositivos como las gafas de realidad virtual y aumentada, que están ofreciendo nuevas posibilidades para desarrollar nuevas narrativas. En comparación con la tecnología móvil, permiten dejar las manos libres y, en el caso de la realidad aumentada, también permiten moverse con naturalidad por la sala 
creando experiencias inmersivas e innovadoras que lograrán aumentar las audiencias en los museos.

Desde nuestra experiencia con Holomuseum hemos comprobado como los nuevos dispositivos de realidad aumentada son ya una posibilidad real para crear experiencias inmersivas impactantes que permitan relacionarse con los datos digitales al tiempo que se interactúa con los objetos reales y el propio espacio expositivo. Asimismo, hemos comprobado como otros museos se han aproximado al uso de la realidad virtual y aumentada creando experiencias impactantes que utilizan la inteligencia artificial o el teatro inmersivo de una manera efectiva. Demostrando una vez más, que la tecnología per sé no es capaz de dar vida al patrimonio cultural, sino que se deben y pueden desarrollar experiencias que se ajusten a las necesidades de los visitantes, las colecciones y las tecnologías.

\section{Referencias}

Goode, G. B. (23 de agosto de 1895). The Relationships and Responsibilities of Museums. (A. A. Science, Ed.) Science, 2(34), 197-209.

Google Arts and Culture. (21 de 02 de 2018). Rhomaleosaurus: Back to Life in Virtual Reality. Obtenido de https://artsandculture.google.com/exhibit/vQLCsZqTLhHBKA

Heritage in motion. (2017). The Lost Palace, Historic Royal Palaces. Recuperado el 17 de febrero de 2018, de https://heritageinmotion.eu/himentry/slugb8d95e6193daf8032ca31da893c3e59b

Horwitz-Bennett, B. (2010). High-Tech Museums: The Future is Now. Interiors + resources.

Kelly, L. (2013). The Connected Museum in the World of Social Media. En K. D. Schroeder, Museum Communication and Social Media. Ther Connected Museum (págs. 58-60). Routledge.

Mapping Sant Climent de Taüll. (2013). Recuperado el 6 de octubre de 2017, de http://pantocrator.cat/es/

Muñoz, A. (junio de 2017). Holomuseum: una aplicación para crear exhibiciones de realidad aumentada. Recuperado el 14 de febrero de 2018, de https://amunyoz.webs.upv.es/blog/holomuseum-una-aplicacion-para-crear-exhibicionesde-realidad-aumentada/

National Public Radio. (19 de diciembre de 2017). Illinois Holocaust Museum Preserves Survivors' Stories - As Holograms. Recuperado el 20 de enero de 2018, de 
https://www.npr.org/2017/12/19/572068474/illinois-holocaust-museum-preservessurvivors-stories-as-holograms

Osterhout, R. (8 de junio de 2016). AWE Annual AR versus VR debate. (T. Furness, Entrevistador) Augmented World Expo (AWE). Recuperado el 12 de diciembre de 2017, de https://www.youtube.com/watch?v=7P-rQXiyyrQ\&t=1733s

Pavid, K. (14 de octubre de 2016). How to resurrect a sea dragon. Recuperado el 19 de febrero de 2018, de http://www.nhm.ac.uk/discover/how-to-resurrect-a-sea-dragon.html

Richardson, J. (enero de 2017). New Immersive Heritage Experience - The Lost Palace. Recuperado el 17 de febrero de 2018, de https://www.museumnext.com/2017/01/newimmersive-heritage-experience-lost-palace/

Rico, J. C. (2006). Manual práctico de museología, museografía y técnicas expositivas. . Madrid: Sílex.

Santacana, J., \& Hernández, F. X. (2006). Museología crítica. Gijón: Trea.

Sierra, A., Riu-Barrera, E., Sugranyes, T., \& Pluma, J. (2015). Taull1123: Immersive experience in a World Heritage Site (or augmented reality without devices). Museums and the Web 2015. Recuperado el 2 de noviembre de 2016, de https://mw2015.museumsandtheweb.com/paper/taull1123-immersive-experience-in-aworld-heritage-site-or-augmented-reality-without-devices/

Witcomb, A. (2003). Re-Imagining the Museum. Beyond the Mausoleum. Nueva York: Routledge. 